\title{
Cerebral revascularization after the Carotid Occlusion Surgery Study: what candidates remain, and can we do better?
}

\author{
Timothy G. White, MD, ${ }^{1}$ Hussam Abou-Al-Shaar, MD,1 Jung Park, MD, PhD, ${ }^{1}$ Jeffrey Katz, MD, ${ }^{3}$ \\ David J. Langer, MD, ${ }^{2}$ and Amir R. Dehdashti, MD1,2
}

Departments of ${ }^{1}$ Neurosurgery and ${ }^{3}$ Neurology, North Shore University Hospital, Barbara and Donald Zucker School of Medicine at Hofstra Northwell; and 'Department of Neurosurgery, Lenox Hill Hospital, Barbara and Donald Zucker School of Medicine at Hofstra Northwell, Manhasset, New York

OBJECTIVE Cerebral revascularization for carotid occlusion was previously a mainstay procedure for the cerebrovascular neurosurgeon. However, the 1985 extracranial-intracranial bypass trial and subsequently the Carotid Occlusion Surgery Study (COSS) provided level 1 evidence via randomized controlled trials against bypass for symptomatic atherosclerotic carotid occlusion disease. However, in a small number of patients optimal medical therapy fails, and some patients with flow-limiting stenosis develop a perfusion-dependent neurological examination. Therefore it is necessary to further stratify patients by risk to determine who may most benefit from this intervention as well as to determine perioperative morbidity in this high-risk patient population.

METHODS A retrospective review was performed of all revascularization procedures done for symptomatic atherosclerotic cerebrovascular steno-occlusive disease. All patients undergoing revascularization after the publication of the COSS in 2011 were included. Perioperative morbidity and mortality were assessed as the primary outcome to determine safety of revascularization in this high-risk population. All patients had documented hypoperfusion on hemodynamic imaging.

RESULTS At total of 35 revascularization procedures were included in this review. The most common indication was for patients with recurrent strokes, who were receiving optimal medical therapy and who suffered from cerebrovascular steno-occlusion. At 30 days only 3 perioperative ischemic events were observed, 2 of which led to no long-term neurological deficit. Immediate graft patency was good, at 94\%. Long term, no further strokes or ischemic events were observed, and graft patency remained high at $95 \%$. There were no factors associated with perioperative ischemic events in the variables that were recorded.

CONCLUSIONS Cerebral revascularization may be done safely at high-volume cerebrovascular centers in high-risk patients in whom optimal medical therapy has failed. Further research must be done to develop an improved methodology of risk stratification for patients with symptomatic atherosclerotic cerebrovascular steno-occlusive disease to determine which patients may benefit from intervention. Given the high risk of recurrent stroke in certain patients, and the fact that patients fail medical therapy, surgical revascularization may provide the best method to ensure good long-term outcomes with manageable up-front risks.

https://thejns.org/doi/abs/10.3171/2018.11.FOCUS18536

KEYWORDS COSS; Carotid Occlusion Surgery Study; carotid occlusion; cerebral bypass; cerebral revascularization

I NDICATIONS for cerebral revascularization have decreased over the last decade. Its indications fall into 2 main categories: 1) flow augmentation in the setting of ischemia, and 2) flow replacement in the setting of complex cerebral aneurysms or tumors. Flow augmentation has been well studied, with the recent publications of the Carotid Occlusion Surgery Study (COSS), the Japanese Adult Moyamoya Trial, and the Japan Extracranial-Intracranial Bypass Trial (JET) ${ }^{8-10}$ Flow augmentation may be done in the setting of moyamoya disease, a rare steno-occlusive

ABBREVIATIONS ACA = anterior cerebral artery; COSS = Carotid Occlusion Surgery Study; CTA = CT angiography; CVR = cerebrovascular reserve; DAPT = dual-antiplatelet therapy; EDAS = encephaloduroarteriosynangiosis; ICA = internal carotid artery; $M C A=$ middle cerebral artery; NOVA = noninvasive optimal vessel analysis; OEF = oxygen extraction fraction; QMRA = quantitative MRA; STA = superficial temporal artery; STLCOS = St. Louis Carotid Occlusion Study; TIA = transient ischemic attack. SUBMITTED October 1, 2018. ACCEPTED November 26, 2018. INCLUDE WHEN CITING DOI: 10.3171/2018.11.FOCUS18536. 
disease of the intracranial vasculature, or for symptomatic cerebral atherosclerotic stenosis and occlusion. However, historical and recent randomized clinical trials have whittled down the indications for bypass in the setting of cerebrovascular atherosclerotic steno-occlusive disease.

The first trial, published in the New England Journal of Medicine in 1985, showed no benefit for cerebral revascularization in patients with symptomatic nonmoyamoya vasculopathy; however, it failed to stratify patients by risk in order to determine which would receive the greatest benefit from intervention. ${ }^{1}$ Subsequently, the St. Louis Carotid Occlusion Study (STLCOS) was able to identify patients at the highest risk for recurrent stroke in the setting of cerebrovascular stenosis and occlusion by using PET oxygen extraction fraction $(\mathrm{OEF}))^{3,5}$ The COSS was then designed on this premise. ${ }^{7}$ In order to determine whether patients with symptomatic cerebral atherosclerotic stenosis and occlusion who are at the highest risk of recurrent stroke would benefit from revascularization, patients in the COSS underwent PET OEF in order to stratify them by risk. They were then randomized to best medical therapy versus surgical revascularization. Despite numerous criticisms, the ultimate outcome showed that surgical revascularization provided no benefit over best medical therapy, mostly due to the impressive natural history of medical therapy and the perioperative surgical risk associated with revascularization. ${ }^{6,11}$ That being said, more recent studies still demonstrate that misery perfusion is a predictor of stroke risk in patients with symptomatic atherosclerotic cerebrovascular steno-occlusive disease, even with newer optimal medical management. ${ }^{13}$

A small group of patients develop ischemic symptoms and symptomatic hypoperfusion (limb shaking and/or crescendo transient ischemic attacks [TIAs] or orthostatic hypoperfusion) even on optimal medical therapy, due to severe hemodynamic impairment. This group of patients has not been well studied by prior trials. There is little literature on their clinical syndrome and subsequent outcome and surgical risk after undergoing revascularization. The purpose of this study was to identify this group of patients and discuss their surgical outcomes in a single institution's series.

\section{Methods}

A retrospective chart review of all patients who underwent cerebral revascularization for symptomatic cerebral atherosclerotic stenosis and occlusion between January 2011 and August 2018 was done. All patients who underwent cerebral revascularization for nonmoyamoya vasculopathy were included in the study. This study was approved by the IRB at the Feinstein Institute under the Northwell Health System. Clinical and demographic data were collected for each patient. Variables studied included patient age, sex, diagnosis, medical history, clinical course, medical therapy, preoperative imaging, postoperative imaging, and postoperative clinical outcomes. Independent radiologists determined bypass patency. Clinical outcomes were gathered from date of last patient follow-up in the medical record. Of particular concern was the perioperative morbidity associated with individual cases; therefore, 30-day outcomes were collected as the primary surgical outcome in these patients.

\section{Patient Choice and Surgical Technique}

Patients were identified as possible candidates for cerebral revascularization if they showed evidence of atherosclerotic carotid or middle cerebral artery (MCA) stenoocclusive disease and optimal medical therapy had failed, or if they had perfusion-dependent neurological examinations. Optimal medical therapy including dual-antiplatelet therapy (DAPT), tight blood pressure control, glycemic control, and statin use was determined by the stroke neurologist taking care of the individual patients and was documented as part of the data that were collected. Once identified as possible candidates, the patients underwent conventional cerebral angiography for diagnostic purposes, to determine the site of disease, and for presurgical planning. All patients also underwent some form of hemodynamic imaging, including either SPECT with and without Diamox, quantitative MRA (QMRA) noninvasive optimal vessel analysis (NOVA), MR perfusion, or CT perfusion. SPECT was done to determine those patients with impaired cerebrovascular reserve (CVR), and QMRA NOVA was used as an adjunct demonstrating decreased flow in the vascular territory in question.

Surgery was subsequently performed teamed with a trained neuroanesthesiologist and with strict blood pressure control. Direct superficial temporal artery (STA)MCA bypass was preferred in all cases because the goal in each procedure was flow augmentation. Postoperatively, patients were followed after 30 days had passed postdischarge in order to accurately assess perioperative morbidity. Postprocedure bypass patency was assessed using digital subtraction angiography, CT angiography (CTA), or QMRA NOVA.

Perioperative management focused on tight blood pressure controls and adherence to an aspirin regimen as well as patient positioning. During the case, systolic blood pressure was maintained above $120 \mathrm{~mm} \mathrm{Hg}$ during the procedure, especially for induction. Postoperative blood pressure goals depended on graft flow but generally were from 120 to $140 \mathrm{~mm} \mathrm{Hg}$. Patients were placed on aspirin $(81 \mathrm{mg}$ ) prior to the procedure, given aspirin (325 mg) on the night of the procedure, and then continued on $81 \mathrm{mg}$ daily. CTA was done on postoperative day 1 . If any patient developed a new deficit on examination, MRI and QMRA NOVA studies were done to assess for hyperperfusion versus new stroke.

\section{Statistical Analysis}

All data were collected and stored via a REDcap (Research Electronic Data Capture) database, and statistics were analyzed using SPSS and Excel. All variables are expressed as the average $\pm \mathrm{SD}$, and where appropriate $\mathrm{p}=$ 0.05 was used for statistical significance. For descriptive statistics the average was calculated. Univariate (Student t-test and chi-square analysis for continuous variable) and multivariate regression were used to predict factors associated with bypass patency and clinical outcome. 
TABLE 1. Characteristics in 35 patients who underwent revascularization procedures

\begin{tabular}{lc}
\hline \multicolumn{1}{c}{ Characteristic } & Value \\
\hline Age, yrs (range) & $55(22-74)$ \\
\hline Female sex & $54 \%(19 / 35)$ \\
\hline Presentation & $74 \%(26 / 35)$ \\
\hline Recurrent stroke & $11 \%(4 / 35)$ \\
\hline Recurrent TIA & $9 \%(3 / 35)$ \\
\hline Perfusion-dependent neurological exam & $3 \%(1 / 35)$ \\
\hline Hemorrhagic transformation of stroke & $3 \%(1 / 35)$ \\
\hline Headache w/ severe hypoperfusion & \\
\hline Vessel occluded & $31 \%(11 / 35)$ \\
\hline MCA & $49 \%(17 / 35)$ \\
\hline ICA & $20 \%(7 / 35)$ \\
\hline Bilat ICA & $60 \%(21 / 35)$ \\
\hline QMRA NOVA & $100 \%(21 / 21)$ \\
\hline Yes & \\
\hline Low flow in affected vessel & $91 \%(32 / 35)$ \\
\hline SPECT & $81 \%(26 / 32)$ \\
\hline Yes
\end{tabular}

\section{Results}

\section{Patient Demographics}

In total, 36 patients underwent cerebral revascularization for nonmoyamoya cerebrovascular occlusive disease. One of the 36 patients was not included in the study because the cerebrovascular occlusion and subsequent hypoperfusion was secondary to carotid sacrifice during head and neck surgery. The average patient age was 55 years (range 22-74 years). All patients except 1 were on at least single-antiplatelet therapy prior to presentation. Clinical presentation included recurrent strokes, recurrent TIA, perfusion-dependent examination, hemorrhagic transformation of stroke, and headache with severe hypoperfusion. The most common presentation was recurrent stroke in $74 \%(26 / 35)$ of patients, followed by recurrent TIA in $11 \%$ $(4 / 35)$, and perfusion-dependent examination in $9 \%(3 / 35)$. Both hemorrhagic transformation of stroke as well as headache and severe hypoperfusion occurred in 1 patient each. Of note, most patients presented with metabolic syndrome including the combination of hypertension, hyperlipidemia, and insulin resistance. There were 3 unique cases in which patients did not present with classic symptoms of atherosclerosis: one patient with fibromuscular dysplasia, one with sickle cell anemia, and one with antiphospholipid syndrome.

\section{Imaging Characteristics}

All patients were evaluated using multimodal imaging techniques. During the workup, patients underwent MRI followed by vessel imaging. In 57\% (20/35) of patients MRI results demonstrated multiple watershed-area infarcts, which was the most frequent MRI finding, whereas other patients presented with multiple MCA but no wa-
TABLE 2. Perioperative outcomes in 35 patients who underwent revascularization procedures

\begin{tabular}{cc}
\hline Outcome & \multicolumn{1}{c}{ Value or Description } \\
\hline Complications & $11 \%(4 / 35)$ \\
\hline Total w/ ischemia & $9 \%(3 / 35)$ \\
\hline Patient 1 & $\begin{array}{c}\text { Postop day 1: neurological change, found } \\
\text { to have graft occlusion; neurological } \\
\text { improvement by time of discharge }\end{array}$ \\
\hline Patient 2 & $\begin{array}{c}\text { Postop day 2: neurological change, found } \\
\text { to have graft occlusion; patient taken } \\
\text { for revision surgery \& EDS procedure } \\
\text { when unable to reopen bypass }\end{array}$ \\
\hline Patient 3 & $\begin{array}{c}\text { Postop day 3: patient developed a wors- } \\
\text { ening neurological exam, graft found } \\
\text { to be patent; by discharge patient had } \\
\text { improved to neurological baseline }\end{array}$ \\
\hline Seizure & $3 \%(1 / 35)$ \\
\hline Total w/ graft patency & $94 \%(33 / 35)$ \\
\hline Long-term graft patency & \\
\hline Total w/ imaging & $60 \%(21 / 35)$ \\
\hline Patency on imaging & $95 \%(20 / 21)$ \\
\hline Length of follow-up & 23 mos (range 3-84) \\
\hline Long-term clinical outcome & \\
\hline Total w/ follow-up & $80 \%(28 / 35)$ \\
\hline Length of follow-up & 25.5 mos (range 3-84) \\
\hline Seizure complication & $4 \%(1 / 28)$ \\
\hline
\end{tabular}

tershed area strokes. All patients underwent some form of hemodynamic imaging. Most commonly the imaging workup included QMRA NOVA as well as SPECT with and without Diamox. QMRA NOVA was done in $60 \%$ $(21 / 35)$ of patients, and in $100 \%$ (35/35) of those patients flow-limiting stenosis was demonstrated when compared to historical age-based normal values. SPECT was done in $91 \%$ of patients (32/35), and of those patients $81 \%(26 / 32)$ showed decreased CVR when challenged with Diamox. All patients without diminished CVR who received Diamox did not improve with the administration of the drug, and at baseline had severe hypoperfusion on SPECT along with recurrent stroke on optimal medical therapy. Patients were categorized as having internal carotid artery (ICA) steno-occlusion, bilateral ICA steno-occlusion, or MCA steno-occlusion. ICA steno-occlusion was the most common, occurring in $49 \%$ of patients (17/35), followed by MCA steno-occlusion in $31 \%$ (Table 1).

\section{Perioperative Outcomes}

Thirty-day perioperative outcomes were assessed in all patients to accurately determine perioperative morbidity in this theoretically high-risk population. Immediate postoperative graft occlusion occurred in 2 patients; therefore immediate graft patency was $94 \%$ (33/35). Postoperative complications occurred in $11 \%$ of patients $(4 / 35)$. There were 3 cases of postoperative ischemic events ( $9 \%$ of patients), which were permanent in only 1 case. Further description of these 3 events can be seen in Table 2. All events oc- 

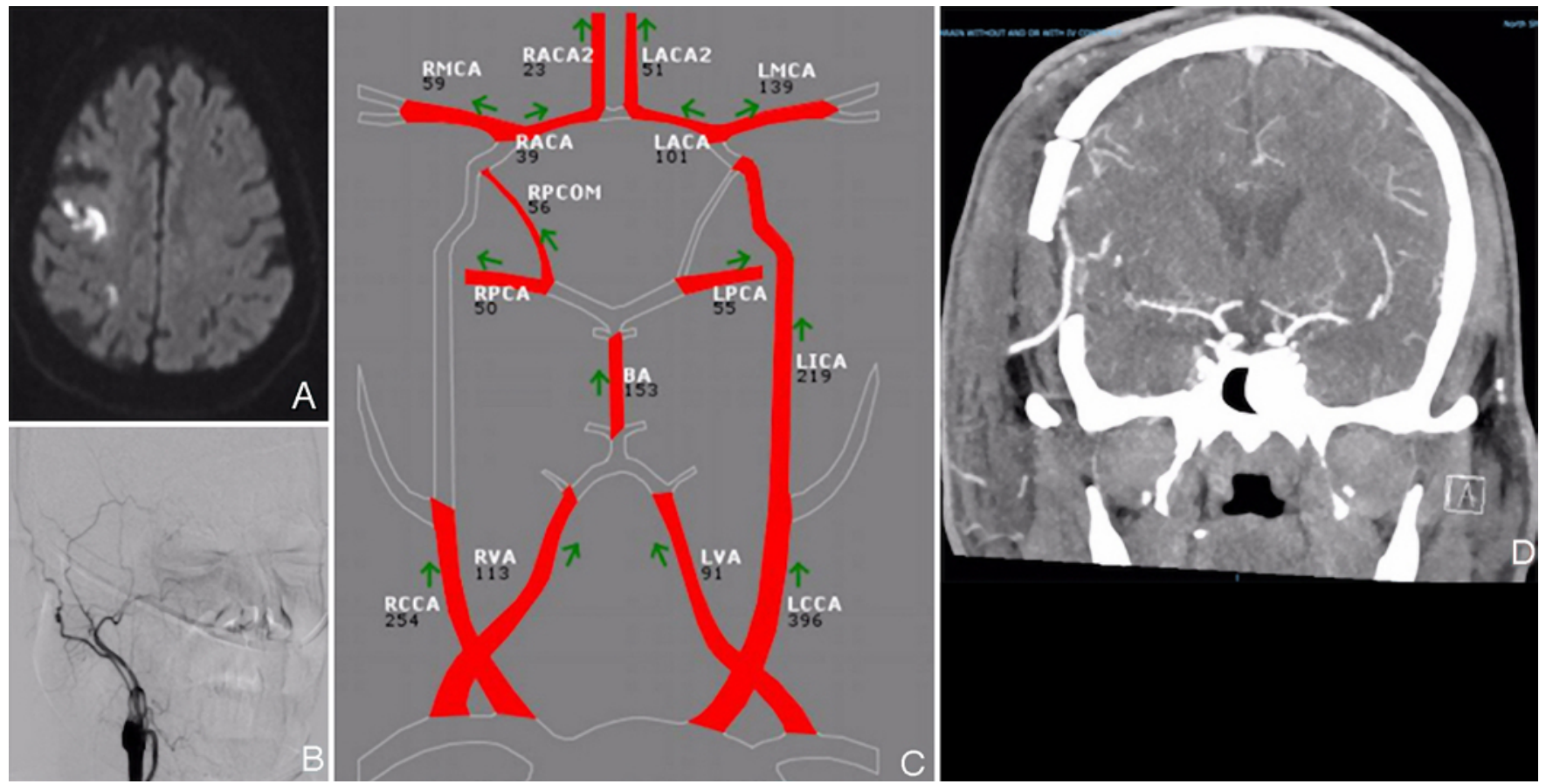

FIG. 1. Case 1. A: MRI, diffusion-weighted imaging (DWI). B: Conventional angiography demonstrating occlusion of the right ICA. C: QMRA NOVA demonstrating right ICA occlusion with a small amount of collateral flow from the right posterior communicating artery. Numbers represent the volumetric flow rate $(\mathrm{ml} / \mathrm{min})$. D: Postoperative CTA showing patent bypass. BA = basilar artery; $L A C A=$ left $A C A ; L C C A=$ left common carotid artery; LICA = left ICA; LPCA = left posterior cerebral artery; $L$ VA = left vertebral artery; RACA = right $\mathrm{ACA} ; \mathrm{RCCA}=$ right common carotid artery; $\mathrm{RPCA}=$ right posterior cerebral artery; $\mathrm{RPCOM}=$ right posterior communicating artery; RVA = right vertebral artery.

curred within 3 days postoperatively. There was also 1 case of postoperative seizure treated successfully with antiepileptic drugs. One patient with postoperative graft occlusion underwent a subsequent encephaloduroarteriosynangiosis (EDAS) procedure when postoperative angiography demonstrated the occlusion. In univariate and multivariate analysis no factors (age, sex, preoperative hemodynamic imaging findings, site of occlusion) were identified as being associated with the risk of a perioperative ischemic event or the likelihood of bypass patency $(\mathrm{p}>0.05)$.

\section{Long-Term Outcomes}

Long-term clinical outcomes were assessed in $80 \%$ (28/35) of patients, whereas long-term graft patency outcomes were assessed in 60\% (21/35) of patients. Bypass grafts were found to be patent in $95 \%$ of patients $(20 / 21)$ (Table 2). In one patient who experienced immediate graft failure on long-term follow-up the graft was found to be open, and in the other patient with immediate occlusion who underwent the revision EDAS procedure long-term patency was not assessed. Clinically, no patient experienced subsequent stroke; however, 1 patient did develop a persistent seizure disorder postoperatively. Duration of follow-up was on average 25.5 months (range 3-84 months).

\section{Illustrative Cases \\ Case 1}

A 58-year-old man with a history of stroke, TIA, hyper- tension, hyperlipidemia, and atrial fibrillation who was on coumadin and DAPT presented to the hospital with left facial droop, hand weakness, and numbness. MRI revealed right MCA territory acute infarcts and angiography demonstrated right ICA occlusion with no filling of the right ICA (Fig. 1A and B). QMRA NOVA demonstrated right ICA occlusion with a small amount of collateral flow from the right posterior communicating artery, and follow-up SPECT with Diamox challenge revealed right-sided hypoperfusion with decreased CVR (Fig. 1C). Given the imaging evidence of right-sided hypoperfusion as well as recurrent strokes on DAPT, a right-sided STA-MCA flow augmentation revascularization was offered and, after a discussion of the risks and benefits, the patient chose to undergo the procedure. Postoperatively the patient experienced no complications, with postoperative imaging showing a patent bypass graft (Fig. 1D). One year after the initial bypass the patient had no further evidence of stroke.

\section{Case 2}

A 60-year-old woman presented with aphasia and right-sided weakness. MRI revealed left-sided basal ganglia and watershed zone infarcts as well as prior watershed ischemic changes (Fig. 2A). CTA and conventional angiography showed $95 \%$ left MCA stenosis, so the patient was placed on an aspirin and Plavix regimen. However, 1 week later she presented back to the hospital with worsening right-sided weakness and recurrence of aphasia after initial improvement from prior admission. Follow-up MRI showed new strokes (Fig. 2B and C). QMRA NOVA 

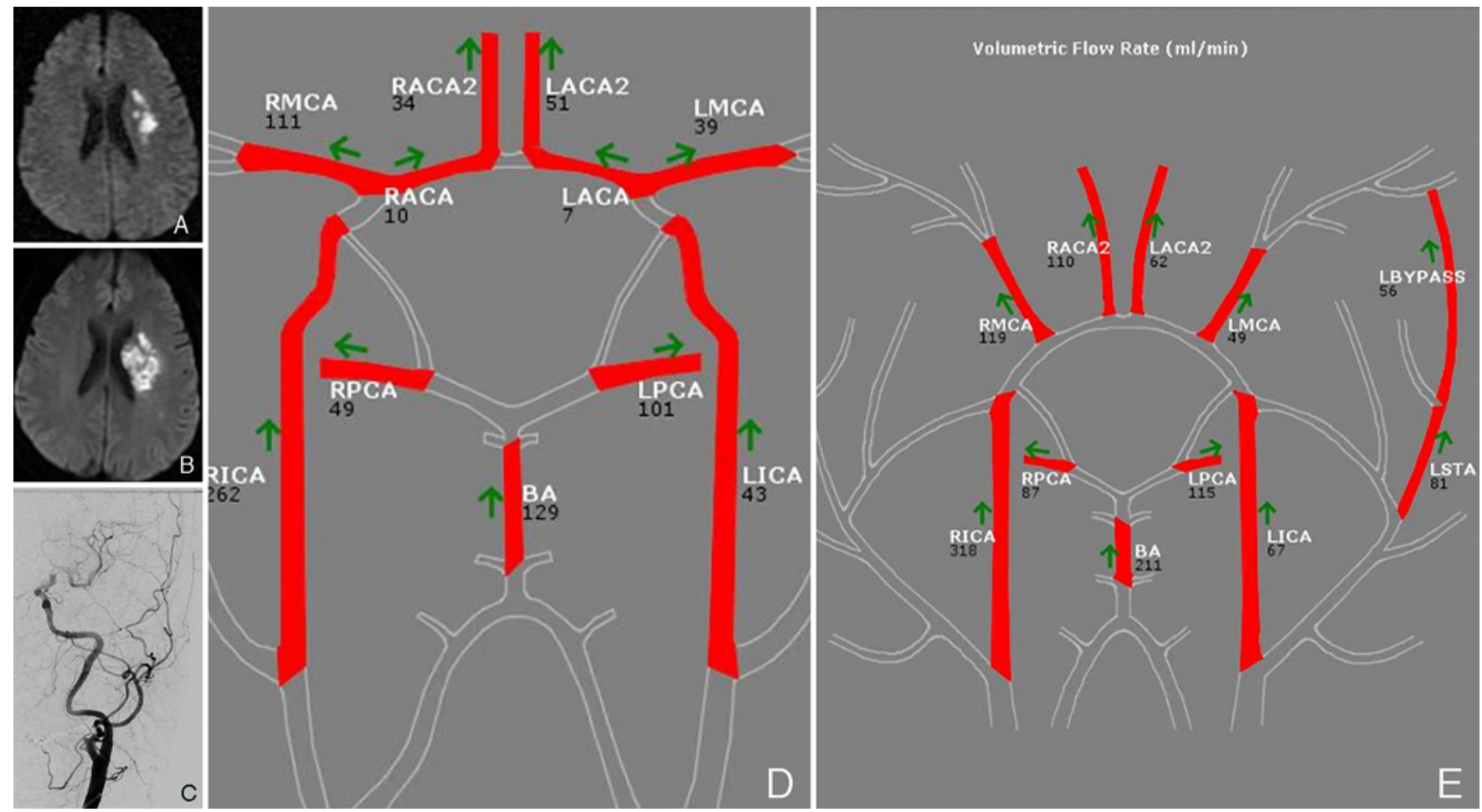

FIG. 2. Case 2. A: Initial MRI, DWI. B: Follow-up MRI, DWI sequence showing progression of stroke. C: Digital subtraction angiography showing severe MCA stenosis. D: Initial QMRA NOVA showing low flow in the left MCA. E: Postbypass QMRA NOVA showing patent bypass with significant flow.

showed low flow in the left anterior cerebral artery (ACA) and left MCA, and SPECT with Diamox showed significant left-sided hypoperfusion with diminished CVR (Fig. 2D). Due to recurrence of stroke and hemodynamic compromise the patient underwent left-sided STA-MCA bypass. Postoperatively the patient experienced no complications and the graft was patent immediately and 1 year postoperatively based on QMRA NOVA (Fig. 2E). At 5 years postbypass the patient had not experienced another stroke.

\section{Discussion}

Prior randomized controlled trials have demonstrated no benefit to surgical revascularization in patients with symptomatic cerebrovascular atherosclerotic steno-occlusive disease. ${ }^{1,10}$ However, optimal medical management, which includes optimization of atherosclerotic and stroke risk factors as well as DAPT, still fails to prevent strokes in a small number of patients. These patients may present with perfusion-dependent examinations or recurrent TIAs and/or strokes. ${ }^{4,11}$ No randomized controlled trial has investigated the role of bypass in this patient group. Although the COSS did stratify patient risk by using hemodynamic imaging modalities, it did not specifically investigate patients in whom best medical therapy failed. ${ }^{4}$ The purpose of this retrospective analysis was to demonstrate the safety and perioperative risks associated with revascularization in theoretically high-risk patients in whom medical management has failed and who showed definitive impaired cerebrovascular reserve. Here we dem- onstrate that when done at a high-volume cerebrovascular center with specified neuroanesthesia, this procedure can be done with a reasonable level of morbidity and may represent an effective salvage procedure in certain patients. In this series perioperative ischemic events occurred in $9 \%$ of patients (3/35), with only 1 patient developing an irreversible neurological deficit.

The original 1985 extracranial-intracranial bypass trial randomized 137 patients with symptomatic carotid or intracranial steno-occlusive disease but failed to stratify patients by risk prior to randomization. Ultimately, no benefit was found with surgical intervention at an average follow-up of 55.8 months, with stroke rates in the medical arm of $29 \%$ versus $31 \%$ in the surgical arm. Following the publication of these results, validated criteria for cerebral hemodynamic impairment were developed via the STLCOS. ${ }^{3,5}$ At that time, studies on bypass hemodynamics demonstrated that revascularization was able to correct impaired hemodynamics in patients. ${ }^{11}$ That being said, the increased OEF used to randomize patients in the COSS may not have been an adequate predictor of hemodynamic impairment. ${ }^{2}$

The STLCOS established that PET OEF was able to predict stroke risk in patients with symptomatic cerebrovascular steno-occlusive disease and stage II hemodynamic impairment. In patients with misery perfusion and increased OEF, the stroke risk approached $26.5 \%$ at 2 years, compared to $5.6 \%$ in patients without impaired perfusion, which led to a predicted benefit of $40 \%$ when developing the COSS and determined patient number to power the study. ${ }^{3,5,7}$ However, in the medical arm of the 
COSS the stroke risk amounted to $22 \%$ over the 2-year study period, with the medical arm faring far better than predicted. ${ }^{10}$ Given that the predicted stroke risk used for the COSS was based on studies done in the 1990s, further studies have investigated whether, with newer medical therapy, misery perfusion still predicts stroke risk. ${ }^{12}$ More recently, Yamauchi et al. demonstrated that misery perfusion on PET OEF still predicts increased stroke risk in patients with symptomatic cerebrovascular steno-occlusive disease. ${ }^{13}$ The authors established that even with the most recent optimal medical management, increased OEF alone (as used in STLCOS and COSS) leaves patients at increased risk of stroke. Therefore, stricter management of these patients is required, and there may still be a role for revascularization in these highest-risk patients. In this study we mainly used SPECT imaging combined with a Diamox challenge in order to perform risk stratification in patients with hypoperfusion and to determine which patients have decreased cerebrovascular reserve.

Ultimately, it was not the goal of this series to validate diagnostic criteria. However, all patients were selected to be possible candidates for bypass based on their clinical presentation combined with hemodynamic imaging in the form of SPECT with and without Diamox, QMRA NOVA, MR perfusion, or CT perfusion. Therefore, further studies would be justified to define the optimal modality to determine impaired cerebral hemodynamics and define misery perfusion.

The outcomes of the COSS were driven by two main factors: the impressively good outcomes of the medically managed patients and the perioperative morbidity of revascularization surgery. Perioperative stroke risk in the COSS was $14 \%$, which was similar to that of the original extracranial-intracranial bypass trial, and these perioperative ischemic events made up the majority of stroke risk in this study group..$^{10}$ Once past the initial perioperative period the surgical patients maintained a significantly lower stroke risk than those in the medical arm. Therefore, decreasing perioperative morbidity and ensuring operative safety is important going forward. In this series we demonstrate that at a high-volume center with specialized neuroanesthesia, perioperative risk in high-risk patients was maintained at less than $10 \%$. Analysis of the COSS data predicted that to show benefit, surgical risk would need to be roughly $50 \%$ of what it was. That being said, this patient population should be considered somewhat separate from the COSS population because, for reasons that have been previously discussed, optimal medical therapy had failed.

Indeed, although the indications for cerebral revascularization for patients with symptomatic steno-occlusive cerebrovascular disease have waned after the publication of the COSS, they have not gone away altogether. However, literature addressing the individual patient populations that may benefit the most has waned as well. This series represents a unique assessment of perioperative risk in high-risk patients not clearly addressed by the COSS. Clearly, further research into this patient group is warranted in order to assess the risk of further stroke after initial therapy as well as to determine optimal treatment modalities.

\section{Conclusions}

Cerebral revascularization for nonmoyamoya symptomatic steno-occlusive cerebrovascular disease has not been shown to provide clinical benefit, but remains an effective and safe option as rescue therapy in patients in whom conventional medical therapy alone has failed. However, this should only be practiced at high-volume centers with specialized staff and experienced surgeons.

\section{References}

1. Barnett HJM, Peerless SJ, Fox AJ, Valberg B, Peacock J, Sackett DL, et al: Failure of extracranial-intracranial arterial bypass to reduce the risk of ischemic stroke. Results of an international randomized trial. N Engl J Med 313:1191-1200, 1985

2. Carlson AP, Yonas H, Chang YF, Nemoto EM: Failure of cerebral hemodynamic selection in general or of specific positron emission tomography methodology?: Carotid Occlusion Surgery Study (COSS). Stroke 42:3637-3639, 2011

3. Derdeyn CP, Yundt KD, Videen TO, Carpenter DA, Grubb RL Jr, Powers WJ: Increased oxygen extraction fraction is associated with prior ischemic events in patients with carotid occlusion. Stroke 29:754-758, 1998

4. Esposito G, Amin-Hanjani S, Regli L: Role of and indications for bypass surgery after Carotid Occlusion Surgery Study (COSS)? Stroke 47:282-290, 2016

5. Grubb RL Jr, Derdeyn CP, Fritsch SM, Carpenter DA, Yundt KD, Videen TO, et al: Importance of hemodynamic factors in the prognosis of symptomatic carotid occlusion. JAMA 280:1055-1060, 1998

6. Grubb RL Jr, Powers WJ, Clarke WR, Videen TO, Adams HP Jr, Derdeyn CP: Surgical results of the Carotid Occlusion Surgery Study. J Neurosurg 118:25-33, 2013

7. Grubb RL Jr, Powers WJ, Derdeyn CP, Adams HP Jr, Clarke WR: The Carotid Occlusion Surgery Study. Neurosurg Focus $14(3): e 9,2003$

8. Kataoka H, Miyamoto S, Ogasawara K, Iihara K, Takahashi JC, Nakagawara J, et al: Results of prospective cohort study on symptomatic cerebrovascular occlusive disease showing mild hemodynamic compromise [Japanese ExtracranialIntracranial Bypass Trial (JET)-2 Study]. Neurol Med Chir (Tokyo) 55:460-468, 2015

9. Miyamoto S, Yoshimoto T, Hashimoto N, Okada Y, Tsuji I, Tominaga T, et al: Effects of extracranial-intracranial bypass for patients with hemorrhagic moyamoya disease: results of the Japan Adult Moyamoya Trial. Stroke 45:1415-1421, 2014

10. Powers WJ, Clarke WR, Grubb RL Jr, Videen TO, Adams HP Jr, Derdeyn CP: Extracranial-intracranial bypass surgery for stroke prevention in hemodynamic cerebral ischemia: the Carotid Occlusion Surgery Study randomized trial. JAMA 306:1983-1992, 2011 (Erratum in JAMA 306:2672, 2011)

11. Reynolds MR, Derdeyn CP, Grubb RL Jr, Powers WJ, Zipfel GJ: Extracranial-intracranial bypass for ischemic cerebrovascular disease: what have we learned from the Carotid Occlusion Surgery Study? Neurosurg Focus 36(1):E9, 2014

12. Yamauchi H, Fukuyama H, Nagahama Y, Nabatame H, Nakamura K, Yamamoto Y, et al: Evidence of misery perfusion and risk for recurrent stroke in major cerebral arterial occlusive diseases from PET. J Neurol Neurosurg Psychiatry 61:18-25, 1996

13. Yamauchi H, Higashi T, Kagawa S, Nishii R, Kudo T, Sugimoto K, et al: Is misery perfusion still a predictor of stroke in symptomatic major cerebral artery disease? Brain 135:25152526, 2012

\section{Disclosures}

The authors report no conflict of interest concerning the materi- 
als or methods used in this study or the findings specified in this paper.

\section{Author Contributions}

Conception and design: all authors. Acquisition of data: White, Abou-Al-Shaar, Park. Analysis and interpretation of data: White. Drafting the article: White. Critically revising the article: White, Dehdashti. Reviewed submitted version of manuscript: White, Langer, Dehdashti. Approved the final version of the manuscript on behalf of all authors: White. Statistical analysis: White. Administrative/technical/material support: Abou-Al-Shaar. Study supervision: Katz, Langer, Dehdashti.

\section{Correspondence}

Timothy G. White: North Shore University Hospital, Barbara and Donald Zucker School of Medicine at Hofstra Northwell, Manhasset, NY.twhite7@northwell.edu. 